\title{
VARIABILITY OF ARTERIAL BLOOD PRESSURE IN CHILDREN WITH DIABETES MELLITUS AND OBESITY \\ Yuriy Nechitaylo ${ }^{1}$, Oleksandr Buriak ${ }^{2}$, Olesya Pidmurniak ${ }^{3}$, Nataliya Kovtyuk ${ }^{4}$, Tatiana Fomina ${ }^{5}$
}

\begin{abstract}
:
INTRODUCTION: The activity of the cardiovascular system has a clear relationship to the circadian rhythms of a child's body. Daily fluctuations in blood pressure in normal conditions and in pathology is a physiological phenomenon which can play a significant role in developing arterial hypertension and even in the occurrence of fatal cardiovascular states such as heart attacks, strokes, and sudden cardiac arrest activities. Ambulatory blood pressure monitoring is recognized as a necessary instrumental investigation for diagnosis and management of patients with arterial hypertension and especially in children from high risk groups such as diabetics and obesity it may be even more important for hypertension diagnostics than in adults.
\end{abstract}

AIM: The objective of the study was to analyze the features of circadian oscillations of blood pressure and heart rate variability in children with diabetes mellitus and hypothalamic syndrome with obesity.

METHODS: Research included 76 children aged 10 to 18 years: 52 children with endocrine pathology (diabetes and obesity) and 24 clinically healthy persons. The peculiarities of nutrition, sleep, and the definition of the chronotype using the HornOstberg questionnaire were carried out. The functional state of the cardiovascular systems was determined by single office measurement and by ambulatory blood pressure monitoring.

FINDINGS AND RESULTS: Ambulatory blood pressure monitoring revealed a number of differences between the groups of children. In diabetic patients, daytime systolic blood pressure was higher with increased variability, while the night blood pressure was significantly lower and with significantly less variability, compared to other groups. Attention is drawn to the higher level of diastolic blood pressure and lower variability at night in obese children.

CONCLUSIONS: Daily fluctuations of arterial pressure in children with diabetes and obesity differ from healthy persons depending on the type of disease and could be regarded as result of circadian biorhythms disruption. In obese persons it appears in the form of nocturnal SBP and DBP elevation but with index of variability compared to the control group. In diabetic children the circadian BP rhythms deviations appear in form of SBP elevation during the day with higher index of its variability. Their BP changes were associated with the presence of diabetic nephropathy and proteinuria.

UDC Classification: 616.12-072.85-053.4, DOI: 10.12955/cbup.v7.1454

Keywords: children, diabetes, obesity, chronotype, blood pressure, ambulatory blood pressure monitoring

\section{Introduction}

Biological rhythms are the result of living creatures' natural adaptation to the environment. Most biorhythms are supported by internal mechanisms, while external periodic factors act as their synchronizers (Marcheva et al., 2013). In scientific research and clinical practice, more than 300 functions of the human body with daily variability are analyzed. The internal biorhythms include the fluctuation of breathing, heart rate, body temperature, digestion, excretory processes, the effectiveness of physical and mental activity etc. (Toh, 2008). The introduction of chronobiology elements into medical practices is associated with the importance of biological rhythms ensuring the normal function of the human body. Disruption of circadian biorhythms result not only in sleep disorders, mental and physical disability, stress tolerance, but also in the development of various diseases (Arble et al., 2015). The activity of the cardiovascular system has a clear attachment to the circadian rhythms of a child's body. At the same time, daily fluctuations in blood pressure (BP) in normal conditions and in pathology are still insufficiently studied physiological phenomenon, which in certain situations can play a significant role even in the occurrence of fatal cardiovascular states such as heart attacks, strokes, sudden cardiac arrest activities, etc.(Correa et al., 2017; DeMarco et al., 2014). At the same

\footnotetext{
${ }^{1}$ Bukovinian State Medical University, Department of Pediatrics, Neonatology and Perinatal Medicine, Chernivtsi, Ukraine, yunech03@yandex.ua

${ }^{2}$ Bukovinian State Medical University, Department of Pediatrics, Neonatology and Perinatal Medicine, Chernivtsi, Ukraine, buriakoleksandr1983@gmail.com

${ }^{3}$ Bukovinian State Medical University, Department of Pediatrics, Neonatology and Perinatal Medicine, Chernivtsi, Ukraine, olesya.pidmurniak@gmail.com

${ }^{4}$ Bukovinian State Medical University, Department of Pediatrics, Neonatology and Perinatal Medicine, Chernivtsi, Ukraine, nkovtyuk@gmail.com

${ }^{5}$ Bukovinian State Medical University, Department of Pediatrics, Neonatology and Perinatal Medicine, Chernivtsi, Ukraine, tetjanafomina79@gmail.com
} 
time, changes in the daily BP levels become pathological in a number of somatic and endocrine diseases and are accompanied by specific effects on the systemic hemodynamics of the body. Among them, diseases such as nephritis, autonomic dysfunction, diabetes mellitus and obesity often lead to biorhythms disruption (Meli et al., 2017; Nijafi et al., 2018). The increasing incidence of obesity is a serious global public health challenge and hypothalamic endocrine disruption during puberty can change hormonally regulated metabolic processes increasing risk of obesity and hypertension. As obesity is a multifactorial and complex endocrine disease, its aetiology involves interactions between genes and the environment and could influence circadian rhythms (Ize-Ludlow et al., 2007; Heindel et al., 2015).

Daily monitoring of ECG and BP are contemporary and accessible methods of non-invasive instrumental diagnostics of cardiovascular system problems, cardiac arrhythmias and conduction disorders, control over the dynamics of changes and assessing the effectiveness of treatment of cardiovascular diseases (Macumber et al., 2016). Analysis of daily changes in BP can be carried out by repeated dynamic measurements or its 24 -hours monitoring. Ambulatory blood pressure monitoring (ABPM) is recognized as a necessary instrumental investigation for diagnosis and management of patients with arterial hypertension (AH) (Delaney et al., 2009). ABPM gives an opportunity to receive information about the level and fluctuations of blood pressure over the daytime, to identify patients with nocturnal hypertension, to identify patients with low or high variability of blood pressure (inadequate or excessive decrease in night hours) and decide on a selection of hypotensive medication, taking into account their effect on BP not only in the daytime, but also at night, to assess their adequacy at lowering blood pressure in response to the drug administration and to select the period of their dosage, (Nijafi et al., 2018). Moreover, the use of ABPM in children may be even more important than in adults for hypertension diagnostics, which is confirmed by studies in recent years (Delaney et al., 2009).

The objective of the study was to analyze the features of circadian oscillations of BP and heart rate variability in children with endocrine pathology - diabetes mellitus and obesity with hypothalamic syndrome.

\section{Materials and methods}

In the study 76 children aged 10 to 18 years: 52 children with endocrine pathology ( 31 children with diabetes, 21 with obesity due to pubertal hypothalamic syndrome) and 24 clinically healthy persons were examined. The groups of children did not significantly differ by age or sex. The peculiarities of nutrition, sleep, the definition of the chronotype using the Horn-Ostberg questionnaire were carried out. The functional state of the cardiovascular systems was determined by BP single office measurement and by ABPM, as well as Holter's daily ECG monitoring. Manual measurements of BP have been used previously but they have the disadvantage of not checking the BP continuously. For the monitoring of ECG and ABPM a cardiomonitoring complex "Rhythm 2000" (NTO Beta, Ukraine) was used. The ABPM system was programmed to measure pressure every $30 \mathrm{~min}$ in daytime from 8:00 AM to 10:00 PM and in nighttime every $60 \mathrm{~min}$ from 10:00 PM to 8:00 AM. Participants with average systolic (SBP) or diastolic (DBP) BP above the 95th percentiles by age, gender and height were regarded as hypertensive and persons with BP greater than 90th percentile but less than the 95th percentile were defined as prehypertensive and represented a category of patients at high risk for developing hypertension. The mean SBP and DBP were calculated separately for the 24-hour period and for awake (daytime) and sleep (nighttime) periods. The index of BP nightly decrease was calculated by which children were divided into "dippers" (10-20\%), "non-dippers" (0-10\%), "overdippers" (more than 20\%) and "night-pickers" with BP increase in the night. According to the ECG monitoring heart rate variability and arrhythmias were evaluated. The results were processed statistically for the difference assertion and standard of probability with all p-values being two-tailed and commonly accepted in biological studies, $\mathrm{p}<0.05$ was taken into account.

\section{Results}

The children subdivision by the chronotype indicators were distributed as follows: morning chronotype predominated in children with diabetes and the control group (61.9\%), evening - in children with obesity (61.3\%). The evening chronotype was associated with a shorter duration of sleep during the night - in children with obesity it was $7.8 \pm 0.15$ hours (in the control group and in patients 
with diabetes $-8.2 \pm 0.23$ hours, $\mathrm{p}=0.11$ ). For the compensation of insufficient duration of night-time sleep, a larger percentage of children in this group had daytime napping (47.6\% vs. $32.2 \%$ in diabetic patients). At the same time, short nocturnal sleep leads to a reduction in the duration of the deep sleep phases and could be considered as a risk factor for the development of obesity (DeMarco VG et al., 2014).

The anthropometric parameters in the groups were close but children with hypothalamic syndrome had a lower height $(163.9 \pm 2.4 \mathrm{~cm}$ versus $172.4 \pm 3.2 \mathrm{~cm}$ in patients with diabetes and $168.2 \pm 2.3 \mathrm{~cm}$ in the control group children) and a higher body mass index $\left(24.4 \pm 1.5 \mathrm{~kg} / \mathrm{m}^{2}\right.$ comparing with $20.9 \pm 0.6$ $\mathrm{kg} / \mathrm{m}^{2}$ and $21.2 \pm 0.9 \mathrm{~kg} / \mathrm{m}^{2}$, respectively).

Traditionally, the assessment of hypertension in children has relied on office blood BP measurements. However, this type of measurements may be misleading for the diagnosis of hypertension, due to the white coat and masked hypertension phenomena in children, as observed in adults. In our research ABPM values differ substantially from casual measurements done in the first day of hospitalization. For example first office measurement of SBP in diabetic patients was lower than average level ABPM daytime data $(124.0 \pm 3.2 \mathrm{~mm} \mathrm{Hg}$ against $129.5 \pm 2.0 \mathrm{~mm} \mathrm{Hg})$ and in obese patients it was higher (134.7 $\pm 2.4 \mathrm{~mm} \mathrm{Hg}$ comparing with $128.4 \pm 3.4$ ). The similar difference existed with DBP values in diabetic children $(74.5 \pm 2.2 \mathrm{~mm} \mathrm{Hg}$ against $76.0 \pm 1.6 \mathrm{~mm} \mathrm{Hg}$ ) but in obese persons it was about the same level $-81.7 \pm 2.5 \mathrm{~mm} \mathrm{Hg}$ and $81.1 \pm 2.5 \mathrm{~mm} \mathrm{Hg}$.

ABPM revealed a number of differences between the groups of examined children (Table 1). The daytime BP is not indicative because it depends on many factors: the level of physical activity, number of school lessons, child's emotion, relation between siblings and family members etc. But in children with diabetes diurnal SBP was the highest and significantly differ in comparison with healthy persons. Assessment BP by age-gender-height percentiles revealed in this group 83.3\% persons with elevated SBP and hypertension. DBP in diabetics was a little beat higher than in healthy persons and with $29.0 \%$ patients with validated data over percentiles normative level. Daytime variability of SBP and DBP was significantly higher than in children of the control group.

In children with obesity, daytime SBP was a little higher and DBP significantly differ in comparison with healthy persons. Assessment BP by age-gender-height percentiles revealed in this group 85.7\% persons with elevated SBP and hypertension. DBP in obese persons was higher than in healthy persons and with measurements of $74.1 \%$, patients were over their percentiles normative level. Daytime variability of SBP was significantly higher than in children of the control group.

The night measurements are more valuable for establishing diagnosis of hypertension due to close microenvironmental conditions during the sleep. Nighttime level of SBP and DBP was the highest in obese persons with significant difference in comparison with both other groups $(\mathrm{p}<0.05)$. Furthermore, children of this group were relatively shorter than healthy and diabetic children $(4-8 \mathrm{~cm})$, which influenced the BP assessment by age-sex-height percentiles chart in the direction of lowering the diagnostically significant BP level. Finally, the data set demonstrated a lack of sufficient variability in $\mathrm{BP}$ values in obese persons. BP variability is one of valuable index of circadian fluctuation and biorhythms disruption. Attention is drawn to the higher level of DBP and less of its variability at night in obese children. Obese individuals have selective insulin resistance, which leads to increased sympathetic activity and alteration of vascular reactivity and resultant sodium retention as evidenced by decreased urinary sodium excretion (DeMarco et al., 2014).

Usually normal BP values peak during the day and drop the following night. Through the level of night decrease, a higher percentage of diabetic children met the criterion of "dippers" (77.4\%) and the overall rate of night-time reduction of blood pressure in this group was more than $11 \%$. The level of variability both SBP and DBP was the highest in comparison with other groups. In children with hypothalamic syndrome with obesity a peculiar circadian profile of BP was recorded with a light SBP decrease in night time ("non-dippers") and monotonous DBP without difference between the day and night. In comparison with other groups the daytime SBP variability was higher and the night variability of systolic and diastolic BP was significantly lower. Most of the children of this group met the "non-dippers'" criterion (61.9\%) and the other part of them were the "night-pickers" (fig.1) with higher nocturnal BP and biorhythm disruption between lower heart rate and elevated SBP, which were absent in other groups. 
In the early morning BP values begin to arise rapidly until they reach the values of the day. The time of this increase mostly depend on the children's chronotype. In our obese patients we found out lack of substantial morning increase. This phenomenon, which develops in a very short interval, is of particular importance because it is a period which increases the risk of cardiovascular events.

\begin{tabular}{|c|c|c|c|c|}
\hline & \multirow{2}{*}{ ABPM index } & \multicolumn{3}{|c|}{ Children groups } \\
\hline & & Diabetics & Obese & Healthy persons \\
\hline 1 & Daytime SBP (mm Hg) & $129.5 \pm 2.0^{*}$ & $128.4 \pm 3.4$ & $124.8 \pm 1.8$ \\
\hline 2 & Daytime DBP & $76.0 \pm 1.6$ & $81.1 \pm 2.5^{*}$ & $71.3 \pm 1.7$ \\
\hline 3 & Daytime SBP variability & $14.5 \pm 0.9^{*}$ & $16.7 \pm 1.3^{*}$ & $11.3 \pm 0.6$ \\
\hline 4 & Daytime DBP variability & $17.0 \pm 1.1^{*}$ & $12.1 \pm 1.2$ & $10.0 \pm 0.6$ \\
\hline 5 & Night SBP & $116.5 \pm 2.1$ & $123.6 \pm 1.9^{*}$ & $118.3 \pm 1.6$ \\
\hline 6 & Night DBP & $67.0 \pm 1.1$ & $81.5 \pm 1.2^{*}$ & $68.3 \pm 1.4$ \\
\hline 7 & Night SBP variability & $13.0 \pm 0.7^{*}$ & $9.9 \pm 0.7$ & $11.4 \pm 0.5$ \\
\hline 8 & Night DBP variability & $12.5 \pm 0.8$ & $4.5 \pm 0.6^{*}$ & $9.6 \pm 0.6$ \\
\hline 9 & Night SBP decrease, $\%$ & -11.1 & -3.7 & -5.2 \\
\hline 10 & Night DBP decrease, $\%$ & -11.9 & +0.5 & -4.3 \\
\hline \multicolumn{5}{|c|}{$*$ - probability of difference, $\mathrm{p}<0.05$} \\
\hline
\end{tabular}

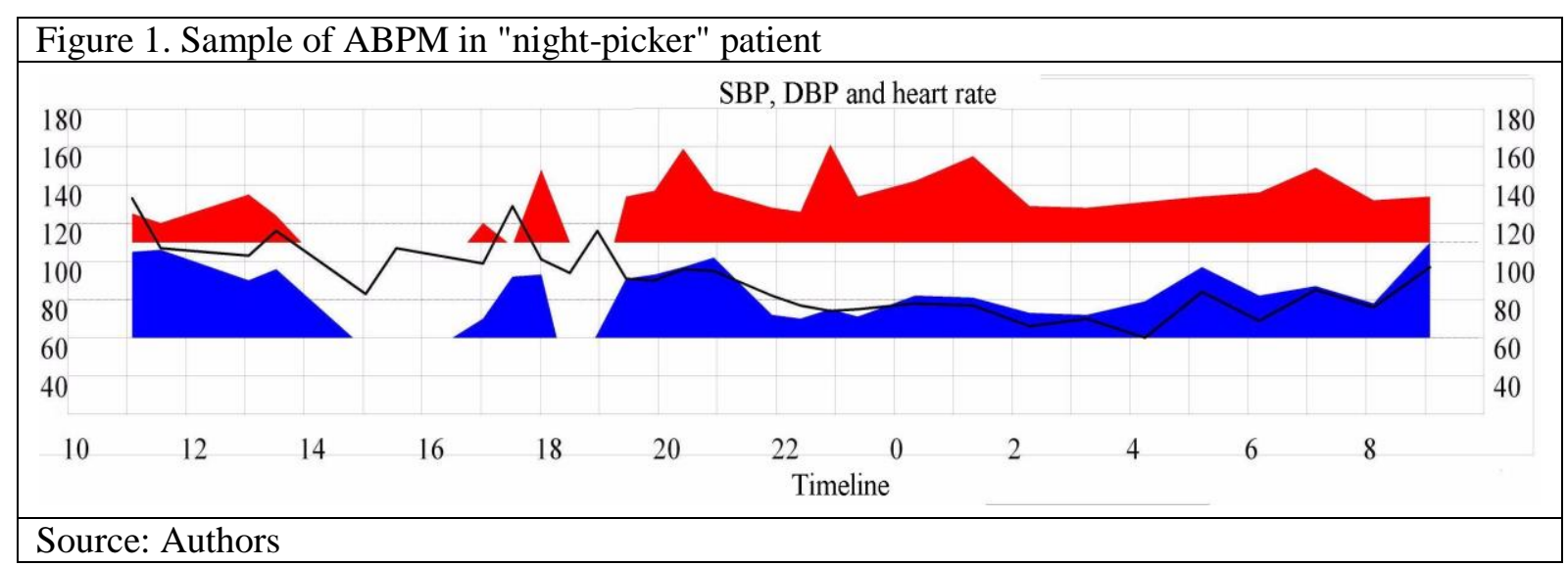

According to the literature data, in overweight children hypertension risk factors are considered a combination of genetic, environmental, behavioral and dietary factors. In our study, we observed a number of correlations: in children with obesity the higher BP was associated with deviations in the regime of daytime activity $(\mathrm{R}=-0.21, \mathrm{p}<0.05)$ and nutrition $(\mathrm{R}=-0.24, \mathrm{p}<0.05)$, imbalance of the diet with predominance of cereal products $(R=-0.24, p<0.05)$, absence of daytime sleep $(R=-0.25, p$ $<0.05)$, increase in BMI $(\mathrm{R}=0.21, \mathrm{p}<0.05)$.

In diabetic patients the causes of BP elevation were mostly associated with target organ damage diabetic nephropathy. We registered in many cases persistent minimal elevation of albuminuria at rest which predicts the development of more severe proteinuria and other clinical symptoms of diabetic nephropathy. Abnormal albumin excretion has been shown to predict the development of clinically significant nephropathy in diabetic patients and presence of angiopathy (Meli et al., 2017). In these patients the nature of the associations was somewhat different from obese persons: increased BP correlates with presence of proteinuria $(r=0.51, p<0.05)$, diet violations $(r=-0.51, p<0.05)$, duration of night sleep $(r=0.24, p<0.05)$, high BMI $(r=0.48, p<0.05)$. Daytime BP variability in diabetic patients are associated with passive smoking at home $(\mathrm{r}=0.45, \mathrm{p}<0.05)$, variability of nocturnal BP correlated with absence of daytime napping $(\mathrm{r}=0.35, \mathrm{p}<0.05)$ and presence and grade of anemia $(\mathrm{r}=$ $-0.39, \mathrm{p}<0.05)$.

\section{Conclusions}

Use of ABPM gives a basis for the diagnosis when there is discordance in BP readings between casual office BP data and mean daytime ambulatory BP measurements. It is particularly useful in patients with white coat and masked hypertension. Diurnal fluctuations of blood pressure in children with 
diabetes and obesity differ from healthy persons depend upon the type of disease and could be regarded as result of circadian biorhythms disruption. In obese persons it appears in the form of nocturnal SBP and DBP elevation but with index of variability compared to the control group. In diabetic children the circadian BP rhythms deviations appear in form of SBP elevation during the day with higher index of its variability. Their BP changes were associated with the presence of diabetic nephropathy and proteinuria.

\section{References}

Arble DM, Bass J, Diniz BC et al. Impact of sleep and circadian disruption on energy balance and diabetes: a summary of workshop discussions. Sleep. 2015 Dec 1; 38(12): 1849-1860. Published online 2015 Dec 1. doi: 10.5665/sleep.5226

Correa CM, Gismondi RA, Cunha AR, Neves MF, Oigman W. Twenty-four hour blood pressure in obese patients with moderate-to-severe obstructive sleep apnea. Arq Bras Cardiol. 2017 Oct; 109(4): 313-320. doi: 10.5935/abc.20170130

Delaney A, Pellizzari M, Speiser PW, Frank GR. Pitfalls in the measurement of the nocturnal blood pressure dip in adolescents with type 1 diabetes. Diabetes Care. 2009 Jan; 32(1): 165-168.doi: 10.2337/dc08-1319

DeMarco VG, Aroor AR, SowersJR. The pathophysiology of hypertension in patients with obesity. Nat Rev Endocrinol. 2014 Jun; 10(6): 364-376. Published online 2014 Apr 15. doi: 10.1038/nrendo.2014.44

Heindel JJ, Newbold R, Schug TT. Endocrine disruptors and obesity. Nat Rev Endocrinol. 2015. 11:653-61. doi: 10.1038/nrendo.2015.163

Ize-Ludlow D, Gray J, Sperling MA, Berry-Kravis EM, Milunsky JM, Farooqi IS, Rand CM, Weese-Mayer DE. Rapid onset obesity with hypothalamic dysfunction, hypoventilation, and autonomic dysregulation presenting in childhood. Pediatrics. 2007;120:e179-e188.

Macumber IR, Weiss NS, Halbach SM, Hanevold CD, Flynn JT. The association of pediatric obesity with nocturnal nondipping on 24-hour ambulatory blood pressure monitoring. Am J Hypertens.2016 May; 29(5): 647-652. doi: 10.1093/ajh/hpv147

Marcheva B, Ramsey KM, Peek CB, Affinati A, Maury E, Bass J. Circadian clocks and metabolism. Handb Exp Pharmacol. 2013;(217):127-55. doi: 10.1007/978-3-642-25950-0_6. Review. PubMed PMID: 23604478.

Meli IHT, Tankeu AT, Dehayem MI, Chelo D, Noubiap JJN. Exercise-induced albuminuria vs circadian variations in blood pressure in type 1 diabetes. World J Diabetes.2017 Feb 15; 8(2): 74-79. Published online 2017 Feb 15. doi: $10.4239 /$ wjd.v8.i2.74

Najafi MT, Khaloo P, Alemi H et al. Ambulatory blood pressure monitoring and diabetes complications: Targeting morning blood pressure surge and nocturnal dipping. Medicine (Baltimore) 2018 Sep; 97(38): e12185. Published online 2018 Sep 21. doi: 10.1097/MD.0000000000012185

Toh KL. Basic science review on circadian rhythm biology and circadian sleep disorders. Ann Acad Med Singapore. 2008 Aug;37(8):662-8. Review. PubMed PMID: 18797559. 